\title{
Cost-effectiveness of biomarker testing for treatment choices in advanced non-small cell lung carcinoma: impact of diagnostic strategies and their turnaround times
}

\author{
To the Editor:
}

Lung cancer remains the main cause of cancer death in the world, not only because of its high frequency but also because it is often diagnosed at poor-prognosis, advanced stages. A better understanding of the molecular mechanisms involved in tumour initiation and progression has permitted to develop new targeted therapies and immunotherapies and molecular testing has now become a standard in care for patients with advanced non-small cell lung cancer (NSCLC). In the era of personalised medicine, the study by Loubière et al. [1] demonstrated that molecular testing prior to treatment initiation is a cost-effective approach in the management of patients with advanced NSCLC. Beside the calculation of global incremental cost-effectiveness ratios, a major interesting point of the study by LouBière et al. [1] is their proposal of a step-by-step evaluation of the costs related to diagnosis, treatments and inpatient care. In this correspondence, I intend to further comment on the diagnosis-related costs and their potential impact on the total costs.

LOUBIÈRE et al. [1] used reference prices for EGFR, KRAS and ALK testing of 180.90, 213.30 and 110.70 EUR per test, respectively. Nevertheless, according to the prices provided by the French Ministry of Health, the costs of molecular analyses can highly vary from one diagnostic strategy to another. For example, to analyse EGFR, 180.90 EUR in fact indicates the price of two-exon analysis, whereas the price of four-exon EGFR analysis (i.e. exons 18-21, as mentioned in the study by Loubière et al. [1]) is 315.90 EUR. A global quote for concomitant EGFR and KRAS analysis in NSCLC is 459.00 EUR but the use of next-generation sequencing (NGS) with a coverage of $<20$ kilobases, commonly used in NSCLC samples for EGFR and KRAS analyses, is set at 882.90 EUR [2,3]. In this manner, it is very difficult to estimate the real costs of molecular analyses per patient in France. In their study, LouBiÈRE et al. [1] highlighted that the diagnosis-related costs (i.e. histopathological diagnosis and biomarker testing) only represented $<3 \%$ (i.e. 513.00-529.00 EUR, including biomarker testing) of the total cost for the management of a patient with NSCLC (i.e. between 17000 and 20000 EUR according to LoUBIÈRE et al. [1]), whereas inpatient care represented between $45 \%$ (biomarker testing strategy) and $68 \%$ (no testing strategy) of the total costs [1]. The diagnosis-related costs could, in this manner, vary from one diagnostic strategy to another but their contribution in the total cost seems to remain minimal, considering the costs of inpatient care and treatments.

Beyond the costs of the molecular tests themselves, various diagnostics methods nevertheless imply different turnaround times (TATs). The TATs from test request to result delivery could, in this manner, in my opinion, strongly influence the cost of the initial management of a patient. Indeed, although the College of American Pathologists, International Association for the Study of Lung Cancer, Association for Molecular Pathology and European external quality assessment schemes have established a benchmark TAT target of 10 working days for the delivery of molecular results to the oncologist, TATs exceeding this

@ERSpublications

Reducing the turnaround times of molecular analyses in patients with advanced lung cancers could help to reduce the costs of the patients' management by permitting rapid treatment choices http://ow.ly/Zv7230jAQv4

Cite this article as: Uguen A. Cost-effectiveness of biomarker testing for treatment choices in advanced non-small cell lung carcinoma: impact of diagnostic strategies and their turnaround times. Eur Respir J 2018; 51: 1800602 [https://doi.org/10.1183/13993003.00602-2018]. 
recommendation are not rare, with some biomarkers results being available only after several weeks for some patients [4-7]. During the time waiting for treatment initiation, the tumours continue to grow and the patients' deterioration could require additional inpatient care, causing increased costs [8]. In this manner, it could be valuable to use first-line fast molecular diagnosis methods able to provide EGFR and KRAS results in a single day (e.g. real-time PCR), as well as those for ALK and now ROS1 and PD-L1 (using dedicated first-line immunohistochemistry) [9, 10]. Such methods could permit rapid treatment choices to optimise the therapeutic management of patients with NSCLC and to reduce inpatient care-related costs during the initial management of patients newly diagnosed with advanced NSCLC. This could be especially relevant for patients' ongoing acute deterioration.

Finally, I fully agree with LoubIÈre et al. [1] that it is likely that, despite the impressive analytical capacities of NGS, clinicians will keep on using a combination of both step-by-step and NGS strategies in the coming years to better match with the management of patients. Beyond expanding the number of molecular analyses (and, to date, expanding their related costs) accompanying the availability of new treatments, the reduction of biomarker analysis TATs could also represent progress for the patients' management, from a medical but also medicoeconomic point of view. This medicoeconomic evaluation of various methods and of the global impact of their TAT on the whole management of patients must be the subject of future studies.

Arnaud Uguen $\oplus^{1,2}$

${ }^{1}$ CHRU Brest, Dept of Pathology, Brest, France. ${ }^{2}$ Inserm U1053 BaRITOn, Bordeaux, France.

Correspondence: Arnaud Uguen, Dept of Pathology, University Hospital Morvan, 2, avenue Foch, 29609 Brest, France. E-mail: arnaud.uguen@chu-brest.fr

Received: March 272018 | Accepted: April 042018

Conflict of interest: None declared.

\section{References}

1 Loubière $\mathrm{S}$, Drezet $\mathrm{A}$, Beau-Faller $\mathrm{M}$, et al. Cost-effectiveness of KRAS, EGFR and ALK testing for decision making in advanced non-small cell lung carcinoma: the French IFCT-PREDICT.amm study. Eur Respir J 2018; 51: 1701467.

2 Ministère des Solidarités et de la Santé 2017 Le Référentiel Des actes innovants hors nomenclature de biologie et d'anatomocytopathologie (RIHN) http://solidarites-sante.gouv.fr/IMG/xlsx/rihn_v2017_20170606.xlsx

3 Ministère des Solidarités et de la Santé. Liste complémentaire d'actes de biologie médical et d'anatomocytopathologie. http://solidarites-sante.gouv.fr/IMG/xlsx/liste-complementaire_v2017_20170607.xlsx. Date last updated: 2017.

4 Lindeman NI, Cagle PT, Aisner DL, et al. Updated molecular testing guideline for the selection of lung cancer patients for treatment with targeted tyrosine kinase inhibitors: guideline from the College of American Pathologists, the International Association for the Study of Lung Cancer, and the Association for Molecular Pathology. Arch Pathol Lab Med 2018; 142: 321-346.

5 van Krieken JH, Siebers AG, Normanno N. European consensus conference for external quality assessment in molecular pathology. Ann Oncol 2013; 24: 1958-1963.

6 Ellis PM, Verma S, Sehdev S, et al. Challenges to implementation of an epidermal growth factor receptor testing strategy for non-small-cell lung cancer in a publicly funded health care system. J Thorac Oncol 2013; 8: 1136-1141.

7 Lim C, Tsao MS, Le LW, et al. Biomarker testing and time to treatment decision in patients with advanced nonsmall-cell lung cancer. Ann Oncol 2015; 26: 1415-1421.

8 O’Rourke N, Edwards R. Lung cancer treatment waiting times and tumour growth. Clin Oncol (R Coll Radiol) 2000; 12: 141-144.

9 Lambros L, Caumont C, Guibourg B, et al. Evaluation of a fast and fully automated platform to diagnose EGFR and KRAS mutations in formalin-fixed and paraffin-embedded non-small cell lung cancer samples in less than one day. J Clin Pathol 2017; 70: 544-549.

10 Ilie M, Butori C, Lassalle S, et al. Optimization of EGFR mutation detection by the fully-automated qPCR-based Idylla system on tumor tissue from patients with non-small cell lung cancer. Oncotarget 2017; 8: 103055-103062.

Copyright (CERS 2018

From the authors:

We would like to thank A. Uguen for his correspondence with comments on our article [1]. In this prospective cohort study, the maximum cost for the diagnosis of advanced non-small cell lung cancer (NSCLC) was estimated to be 620 EUR, including EGFR/KRAS/ALK testing. In $52 \%$ of the patients in the "at least one biomarker status known", the diagnostic strategy included EGFR/KRAS and ALK testing 
simultaneously, incurring a mean cost around 550 EUR. In $32 \%$ of the "at least one biomarker status known" group, the diagnostic strategy consisted first of a molecular analysis for KRAS with simultaneous immunohistochemistry (IHC) ALK testing, and second a targeted analysis for EGFR, followed by an additional validation by fluorescence in situ hybridisation assay for ALK IHC positive cases, for example at the time of second-line treatment initiation. For those patients, the total cost of the diagnostic strategy was estimated to be 616 EUR. All costs were based on reimbursed prices provided by the French Ministry of Health.

Depending on the point of view adopted, be it societal, healthcare payer or healthcare provider, the type and level of accuracy for cost estimates are not the same [2]. From the healthcare payer perspective we adopted in our study, using prices to assess diagnosis costs is the recommended approach [2, 3]. Nonetheless, from the point of view of the healthcare provider (i.e. hospital), we acknowledge the inadequacy of using prices rather than accurate costs, and as reported by A. Uguen as well, the difficulty in capturing such costs. For example, there is limited national data on the magnitude of each component of those biomarker testing costs, such as labour (including but not limited to technician/engineer mobilised during all the steps and biologist or pathologist time), consumables, and equipment and overhead costs. Thus, more reliable estimates of diagnosis costs would be useful inputs for economic evaluation of strategies based on specific biomarker testing with appropriate targeted or non-targeted therapies from the providers' perspective. Therefore, we are conducting a micro-costing analysis on the costs of each technology of testing used in the IFCT-PREDICT.amm study in order to generate accurate cost estimates for biomarker testing strategies through a detailed measurement and valuation of resource inputs. In addition, we are conducting the same analysis on next-generation sequencing (NGS) techniques performed in the same certified genetic centres. We favoured a micro-costing approach, which is particularly well-suited to capturing the variations between settings and patients, as the total costs of EGFR and KRAS mutations testing (including DNA extraction) are expected to vary across French National Cancer Institute-certified genetic centres according to the different mutation analysis strategies.

Considering the remark of A. Uguen on the turnaround time (TAT), we acknowledge that the risk inherent to a step-by-step approach for the NSCLC diagnosis would be an increased proportion of patients displaying an unknown oncogenic driver prior to starting first-line treatment, due to excessive TAT to obtain the second set of molecular analyses. The IFCT Biomarker France study showed that $23 \%$ of NSCLC patients had started treatment before they received the results of the molecular analysis [4]; the main reason being the too long TAT of biomarker results in those specific cases. In a retrospective study conducted in Canada between April 2010 and March 2013, the prevalence of biomarker testing rose from $46 \%$ in the first year to $60 \%$ during the last year of the study for EGFR testing, and from $5 \%$ to $40 \%$ for ALK testing, with $19 \%$ of patients having started chemotherapy before their biomarker results became available [5]. These results are very similar to those found in our study.

Although the small panel NGS recently implemented in France allows simultaneous analysis of several molecular targets (EGFR, HER2, BRAF, etc.), it does not allow identification of the presence of gene rearrangements, such as ALK or ROS [6]. Moreover, the new targetable alterations mainly concern rearrangements (RET, NTRK, NRG1, NTRK, etc.) or complex alterations such as the skip exon 14 mutation of MET which also require the concurrently use of several techniques. Finally, the small panel NGS currently used does not measure the mutation burden of the tumour, which appears to be an emerging biomarker for the prescription of immunotherapies. In the coming years, it is likely that several diagnostic techniques will still be required to examine all predictive biomarkers in thoracic oncology, always asking the question of the step-by-step approach and risking further lengthening the TAT. To conclude, it is hoped that in future, though the timeframe is uncertain, RNA or exome sequencing approaches with hybrid capture will make all predictive biomarkers immediately available before starting first-line treatment at reasonable cost. The Biomarkers France \#2 study is being initiated precisely in order to capture the changes that occurred with the implementation of NGS and includes more than two-thirds of the advanced NSCLC patients tested in 2017.

@ERSpublications

Costs and turnaround-time impact of biomarker testing for treatment choices in advanced NSCLC in the NGS era http://ow.ly/16BV30k8Soz

Cite this article as: Loubière S, Barlesi F, Missy P, et al. Cost-effectiveness of biomarker testing for treatment choices in advanced non-small cell lung carcinoma: impact of diagnostic strategies and their turnaround times. Eur Respir J 2018; 51: 1800844 [https://doi.org/10.1183/13993003.00844-2018]. 
Sandrine Loubière ${ }^{1,2}$, Fabrice Barlesi ${ }^{3}$, Pascale Missy ${ }^{4}$, Franck Morin ${ }^{4}$, Pascal Auquier ${ }^{1,2}$ and Jacques Cadranel ${ }^{5}$ ${ }^{1}$ Assistance Publique Hôpitaux de Marseille, DRCI, Service Epidémiologie et Economie de la Santé, Marseille, France. ${ }^{2}$ Aix Marseille Univ, Faculté de Médecine - Secteur Timone, EA3279: CEReSS - Centre d’Etude et de Recherche sur les Services de Santé et la Qualité de vie, Marseille, France. ${ }^{3}$ Université Aix Marseille, Assistance Publique Hôpitaux de Marseille, Département Oncologie multidisciplinaire et innovations thérapeutiques, Hôpital Nord, Centre d'investigation clinique, Marseille, France. ${ }^{4}$ Intergroupe Francophone de Cancérologie Thoracique (IFCT), Unité de recherche clinique, Paris, France. ${ }^{5}$ Assistance Publique Hôpitaux de Paris, Hôpital Tenon, Service de Pneumologie, Sorbonne Universités, UPMC Univ Paris 06, GRC n04, Theranoscan, Paris, France.

Correspondence: Sandrine Loubiere, APHM, EA3279 - CEReSS, Faculté de Médecine Timone, 27 Boulevard Jean Moulin, F-13005 Marseille Cedex 5, France. E-mail: sandrine.loubiere@univ-amu.fr

Received: May 072018 | Accepted: May 192018

Conflict of interest: F. Barlesi reports personal fees from AstraZeneca, Bristol-Myers Squibb, Boehringer Ingelheim, Clovis Oncology, Eli Lilly Oncology, F. Hoffmann-La Roche Ltd, Novartis, Merck, MSD, Pierre Fabre and Pfizer, outside the submitted work. J. Cadranel reports grants for research on ALK immune response and personal fees for participation on advisory boards for clinical trials from Pfizer, grants for research on NGS and personal fees for participation on advisory boards for clinical trials from Novartis and AstraZeneca, and personal fees for participation on advisory boards for clinical trials from Roche, Boehringer Ingelheim, BMS and Lilly, outside the submitted work.

\section{References}

1 Loubière S, Drezet A, Beau-Faller M, et al. Cost-effectiveness of KRAS, EGFR and ALK testing for decision making in advanced non-small cell lung carcinoma: the French IFCT-PREDICT.amm study. Eur Respir J. 2018; 51: 1701467.

2 Husereau D, Drummond M, Petrou S, et al. Consolidated Health Economic Evaluation Reporting Standards (CHEERS)-explanation and elaboration: a report of the ISPOR Health Economic Evaluation Publication Guidelines Good Reporting Practices Task Force. Value Health 2013; 16: 231-250.

3 Haute Autorité de Santé. Guide Méthodologique: Choix méthodologiques pour l'évaluation économique à la HAS. Octobre 2011, Edition HAS, service communication. Saint-Denis La Plaine, Haute Autorité de Santé, 2011. Available from: www.has-sante.fr/portail/upload/docs/application/pdf/2011-11/guide_methodo_vf.pdf

4 Barlesi F, Mazieres J, Merlio JP, et al. Routine molecular profiling of patients with advanced non-small-cell lung cancer: results of a 1-year nationwide programme of the French Cooperative Thoracic Intergroup (IFCT). Lancet 2016; 387: 1415-1426.

5 Lim C, Tsao MS, Le LW, et al. Biomarker testing and time to treatment decision in patients with advanced nonsmall-cell lung cancer. Ann Oncol 2015; 26: 1415-1421.

6 Heeke S, Hofman V, Long-Mira E, et al. Use of the ion PGM and the GeneReader NGS systems in daily routine practice for advanced lung adenocarcinoma patients: a practical point of view reporting a comparative study and assessment of 90 patients. Cancers 2018; 10: E88. 